\section{Grumpy old ethicist}

\section{Derek Willis}

I am aware that I have picked two strands of Medicine that seem to have an image problem. As I discussed in the last piece, I deal with patients who I know are not going to get better. The second strand is ethics. Some say that medical ethics is just opinion, and rather than producing anything 'useful', ethics is accused of muddying (already muddy) waters and so, is inherently 'useless'.

Medical ethics is a branch of philosophy, and at its heart, philosophy attempts to describe good ways to live. Over 2000 years of thought have gone into this, and if the conclusions it reaches are complicated, it is because life itself is tricky, messy and complicated. Specifically for doctors, an ethicist would want to say that there is a right way to understand and to practice medicine. An ethicist has a responsibility to struggle, discuss and work out what that right way should look like. To help healthcare professionals be 'good' healthcare professionals is at the purpose of good medical ethics in practice.

Now, I think that we ethicists probably do have some responsibility for the poor image, and I would want to say that some of this is down to how ethicists teach the subject. It would be unusual to find a teaching

Correspondence to Dr Derek Willis, Severn Hospice, Telford TF1, UK;

derekwillis35@hotmail.com session involving medical ethics where the four pillars of ethics are not quoted. As you will know, if you have attended one of these sessions; the four pillars are autonomy, nonmaleficence, beneficence and justice. The four pillars stand on the foundation of a theory known as principlism.

All four principles are equally important, and none of them has prominence over the others. And yet, we often seem to imply that autonomy is the most important. This is not the case. All four are meant to be a check and a balance to each other, so you cannot have one without the others. However, in an individualistic Western culture more emphasis is given to autonomy, especially the autonomy of the specific patient in front of us.

I think we ethicists are also guilty of a worse sin. I think that the way that we teach these principles is a kind of prescription ethics, akin to moral algebra. It is as if we tell our students that if we take our moral problem and enter the facts of the problem into the four principles, somehow magically we will end up with our moral answer. The truth is we do not end up with any answer at all; we end up with more questions. So we then get the reputation of not providing answers, just a whole bunch of more moral confusion.

The truth is; the four principles were never designed to answer questions but were meant to produce a model through which people who held diametrically opposed positions could start a conversation. If we hold different views about what constitutes a moral action then we can never start talking about possible answers. If we start from different positions, we have no common language and no hope of sharing a conclusion.

The principles are tools through which we can start asking questions that we can all agree on, an attempt for us to find some common ground to start talking from. Once we start asking questions together we can start addressing the particulars about the specifics of clinical situations. To put this even more simply the four principles cause us to ask rather than answer questions because that is what they were designed to do. They are meant to be a starting point for a discussion rather than a destination.

Some suggest that the four principles should be put to rest and not taught. I am not sure I would go that far, but we need to be clear about what we mean and what the words that we use are for. We also need to make clear what the point of asking difficult questions is, if we want to avoid having an image problem, and if we want to nurture philosophy (the love of wisdom) within the practice of palliative care.

\section{Competing interests None.}

Provenance and peer review Not commissioned; internally peer reviewed.

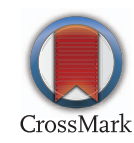

To cite Willis D. BMJ Supportive \& Palliative Care 2015;5:99.

Accepted 13 January 2015 